\title{
THE
}

\section{Enhancement of macrophage uptake via phosphatidylserine- coated acetalated dextran nanoparticles}

\author{
Nishan K. Shah \\ University of Rhode Island \\ Sweta K. Gupta \\ University of Rhode Island \\ Zimeng Wang \\ University of Rhode Island \\ Samantha A. Meenach \\ University of Rhode Island, smeenach@uri.edu \\ Follow this and additional works at: https://digitalcommons.uri.edu/che_facpubs \\ Part of the Chemical Engineering Commons
}

The University of Rhode Island Faculty have made this article openly available.

Please let us know how Open Access to this research benefits you.

This is a pre-publication author manuscript of the final, published article.

Terms of Use

This article is made available under the terms and conditions applicable towards Open Access Policy Articles, as set forth in our Terms of Use.

\section{Citation/Publisher Attribution}

Shah, N. K., Gupta, S. K., Wang, Z., \& Meenach, S. A. (2019). Enhancement of macrophage uptake via phosphatidylserine-coated acetalated dextran nanoparticles. Journal of Drug Delivery Science and Technology, 50, 57-65. doi: 10.1016/j.jddst.2019.01.013

Available at: https://doi.org/10.1016/j.jddst.2019.01.013 


\section{Enhancement of Macrophage Uptake via Phosphatidylserine-Coated Acetalated} Dextran Nanoparticles

\author{
Nishan K. Shah ${ }^{1}$, Sweta K. Gupta ${ }^{2}$, Zimeng Wang ${ }^{2}$, Samantha A. Meenach ${ }^{1,2, *}$ \\ ${ }^{1}$ University of Rhode Island, College of Pharmacy, Department of Biomedical and \\ Pharmaceutical Sciences, Kingston, RI 02881 \\ ${ }^{2}$ University of Rhode Island, College of Engineering, Department of Chemical \\ Engineering, Kingston, RI 02881
}


32 Fax: 401.874 .4689

33

34 Abstract

35 Although vital to the immune system, macrophages can act as reservoirs for 36 pathogens such as tuberculosis and human immunodeficiency virus. Limitations in the

37 treatment of such diseases include targeting therapeutics directly to macrophages and the

38 large systemic dosages needed. The objective of this study is to develop a nanoparticle

39 (NP)-based drug delivery system that can provide targeted delivery into macrophages.

40 Acetalated dextran (Ac-Dex) NP loaded with the lipophilic model compound curcumin

41 (CUR) were synthesized and coated in 1,2-dipalmitoyl-sn-glycero-3-phospho-L-serine

42 (DPPS), a phospholipid that induces phagocytosis in macrophages. DPPS-CUR NP were

43 found to release $67.8 \%$ of encapsulated CUR within 24 hours at pH 5.35 and exhibited

44 minimal CUR release $(6.3 \%)$ at $\mathrm{pH}$ 7.4. DPPS-CUR NP were uptaken by murine

45 macrophages significantly more than NP without DPPS coating and NP exposure to these

46 macrophages resulted in minimal toxicity to the cells and minimal nitric oxide production.

47 These results suggest that the combination of the DPPS coating and $\mathrm{pH}$-sensitive polymer

48 Ac-Dex can provide a NP delivery system capable of enhanced uptake by macrophages

49 and potential systemic stability to more effectively deliver drugs of interest. As a result,

50 the described DPPS-CUR NP can serve as a viable delivery system for the treatment of

51 macrophage-associated diseases.

52

53

54

55 


\section{Keywords}

59 Acetalated dextran; 1,2-dipalmitoyl-sn-glycero-3-phospho-L-serine (DPPS); macrophage-

60 associated diseases; targeted cellular uptake; nanoparticles; drug delivery

\section{Abbreviations}

63 2-methoxypropene (2-MOP), acetalated dextran (Ac-Dex), curcumin (CUR), cyclic-to64 acyclic ration (CAC), dichloromethane (DCM), dimethyl sulfoxide (DMSO), deionized 65 (DI) water, 1,2-dipalmitoyl-sn-glycero-3-phosphocholine (DPPC), 1,2-dipalmitoyl-sn66 glycero-3-phospho(1'-rac'glycerol) (DPPG), 1,2-dipalmitoyl-sn-glycero-3-phospho-L67 serine (DPPS), differential scanning calorimetry (DSC), encapsulation efficiency (EE), 68 human immunodeficiency virus (HIV), lipopolysaccharide (LPS), nitric oxide (NO), 69 nanoparticles (NP), phosphate buffered saline (PBS), phosphatidylserine (PS), poly(vinyl 70 alcohol) (PVA), poly(lactic-co-glycolic acid) (PLGA), p-toluenesulfonate (PPTS),

71 scanning electron microscopy (SEM), tuberculosis (TB), transmission electron microscopy

72 (TEM), triethylamine (TEA)

73 


\section{Introduction}

75 Macrophages are vital in the removal of cellular debris and foreign bodies to

76 maintain homeostasis in the human body [1,2], can colonize in the liver, lungs, spleen,

77 lymph nodes, marrow, or brain, and are critical to the innate immune system [2]. An

78 example of the importance of macrophages is in the removal of apoptotic cells via

79 stimulation of various signals and markers that are overexpressed by presenting cells to

80 initiate identification and engulfment [3]. Although macrophages are involved in the

81 protection and maintenance of the human body, there are scenarios in which these cells can

82 result in more harm than good. Macrophages have the potential to act as reservoirs for

83 infectious pathogens, including those related to two of the most prevalent infectious

84 diseases, tuberculosis (TB) [1,2] and the human immunodeficiency virus (HIV) [1,2]. The

85 commonality amongst these diseases lies in the extensive treatment times and dosing

86 regimens necessary to treat $\mathrm{TB}$ and $\mathrm{HIV}$, often resulting in negative side effects. As a result,

87 treatment by way of enhanced delivery to macrophages is of growing interest [1-17].

88 Recent progress made in the development of macrophage targeting systems have

89 been based around receptor-ligand interactions [18]. One common approach is surface

90 functionalization of particle systems with mannose residues to target the mannose receptor

91 CD206), a carbohydrate-recognition domain that is largely expressed on alveolar

92 macrophages $[19,20]$. In other cases, tumor associated macrophages have been targeted

93 using peptide-based ligands such as rabies virus glycoprotein (RVG), and can be used as

94 carriers for systems encapsulating anti-cancer therapies [21,22]. Other ligands such as 4-

$95 \mathrm{SO}_{4}$-GalNAc have been used to target other receptors on the surface of phagocytic

96 macrophages to increase targeted uptake [23]. 
Phosphatidylserine (PS) is an anionic phospholipid that is produced and stationed

98 on the inner membrane of healthy cells $[3,5,24,25]$. Once apoptosis is induced, PS

99 transitions from the inner leaflet to the outer leaflet of the cell membrane [14]. Apoptotic

100 cells produce a signal via PS exposure to stimulate the attraction of macrophages for

101 engulfment of the presenting cells (phagocytosis) via receptor-ligand interactions or

102 identification of PS binding proteins produced by phagocytes $[1,3,25,26]$. Although it is

103 essential for the phagocytosis signal, there is some debate as to whether sole exposure of

104 PS is sufficient to induce the uptake of apoptotic cells by macrophages in vitro $[3,25,26]$.

105 However, particle-based drug delivery systems that utilize PS (specifically, 1,2-

106 dipalmitoyl-sn-glycero-3-phospho-L-serine, DPPS) as the particle coating have resulted in

107 enhanced macrophage uptake through the sole presence of PS [3], likely due to the amount

108 of PS presented. Multiple studies centered around treatment of inflammation [7,27], HIV-

$1091[1,2,6,17]$, cancer [8,9,28], atherosclerosis [29], and MRI imaging [1,11,16] have used

110 DPPS coatings to increase the uptake of particle-based delivery systems by macrophages

111 or have used PS as a binding target.

112 Acetalated dextran (Ac-Dex) is a biodegradable, biocompatible polymer comprised

113 of the FDA-approved excipient dextran and hydrophobic acetal groups that allow for

114 emulsion-based NP synthesis [30-32]. Unlike commonly used drug carriers such as

115 poly(lactic-co-glycolic acid) (PLGA), the degradation kinetics of Ac-Dex can be easily

116 tuned by altering the ratio of cyclic to acyclic acetal groups by modifying the Ac-Dex

117 synthesis reaction time [33,34]. The acid-sensitivity of Ac-Dex has made it a viable carrier

118 option in applications for cancer [33-35], inflammatory-related conditions [36], vaccines

119 [33,34], and antibiotic delivery [37]. There is a significant difference in degradation and 
120 drug release kinetics for Ac-Dex nanoparticle (NP) systems at a $\mathrm{pH}$ of 5 (faster release)

121 versus at a pH of 7.4 (slower release) [4,30,31,33-37]. Due to this difference, Ac-Dex NP

122 systems will exhibit slower release and stability during systemic circulation (at $\mathrm{pH}$ 7.4)

123 until they are delivered to a site in the body with a lower $\mathrm{pH}$, such as in macrophages,

124 where therapeutics will be readily released.

125 The current study involves the design of a novel therapeutic particle-based drug

126 delivery system consisting of a phosphatidylserine (PS)-coated, polymeric (Ac-Dex), drug-

127 loaded NP designed to enhance uptake into macrophages. The novelty of this formulation

128 lies in the inherent ability of PS to enhance the uptake of the NP into macrophages, while

129 the Ac-Dex core promotes burst release of the cargo directly in the macrophages owing to

130 its acid sensitivity. Curcumin (CUR), a natural product isolated from Curcuma longa, was

131 chosen as the model small molecule due to its fluorescent properties, low water solubility, 132 and potential clinical applications $[4,5,7,9,13,30,38-42]$.

133

\section{Materials and Methods}

135

136 Materials

137 Unless stated otherwise, materials were purchased from Sigma Aldrich (St. Louis, 138 MO). 1,2-dipalmitoyl-sn-glycero-3-phosphocholine (DPPC), 1,2-dipalmitoyl-sn-glycero139 3-phospho(1'-rac'glycerol) (DPPG), and 1,2-dipalmitoyl-sn-glycero-3-phospho-L-serine

140 (sodium salt, DPPS) were purchased from Avanti Lipids (Alabaster, AL).

142 Synthesis and Characterization of Acetalated Dextran 
144 [30,31]. $1 \mathrm{~g}$ of lyophilized dextran $(9,000-11,000 \mathrm{MW})$ was dissolved in $10 \mathrm{~mL}$ of

145 anhydrous DMSO with $25 \mathrm{mg}$ of p-toluenesulfonate (PPTS) under nitrogen gas. The

146 reaction was carried out for 5 minutes using $5 \mathrm{~mL}$ of 2-methoxypropene (2-MOP) and was

147 quenched with $1 \mathrm{~mL}$ of triethylamine (TEA). The polymer was then precipitated in basic

148 water ( $\mathrm{pH}$ 9), filtered, lyophilized, and stored at $-20^{\circ} \mathrm{C}$. The cyclic-to-acyclic (CAC) ratio

149 of Ac-Dex and acetal coverage were verified using ${ }^{1} \mathrm{H}$ NMR (Bruker $300 \mathrm{MHz}, \mathrm{NMR}$,

150 MA) as described previously [30,31,37].

151

152 Synthesis of PVA-Coated Nanoparticles

153 PVA-coated, CUR-loaded Ac-Dex NP (PVA-CUR NP) were synthesized using a

154 single emulsion/solvent evaporation method [31]. $50 \mathrm{mg}$ of Ac-Dex and $1 \mathrm{mg}$ of CUR were

155 dissolved in $1 \mathrm{~mL}$ of DCM. This organic solution was added to $6 \mathrm{~mL}$ of $3 \%$ PVA (in $1 \mathrm{x}$

156 PBS) and the resulting mixture was emulsified using a probe sonicator attached to an

157 ultrasonic processer at $120 \mathrm{~W}$ and $20 \mathrm{kHz}$ (Q500, Qsonica, Newtown, CT) for 1 minute

158 with 1 second on/off pulses. The emulsion was added to $40 \mathrm{~mL}$ of $0.3 \%$ PVA and was spun

159 for 4 hours to allow for organic solvent evaporation and particle hardening [30]. The 160 spinning solution was centrifuged at $3124 \mathrm{x} g$ for 60 minutes and particles were redispersed

161 in a $0.1 \%$ PVA solution and frozen overnight, followed by lyophilization. PVA-coated NP

162 without CUR (PVA-Blank NP) were formulated similarly to PVA-CUR NP by omitting

163 CUR from the organic solutions. NP samples were stored at $-20^{\circ} \mathrm{C}$.

164

165 Synthesis of DPPS and DPPC-Coated Ac-Dex NP 
167 make liposomal delivery systems $[6,7,17,27,43,44]$. DPPS was dissolved in $4 \mathrm{~mL}$ of

168 chloroform and methanol $(9: 1 \mathrm{v} / \mathrm{v})$ [9]. The solution was subjected to rotary evaporation

169 using a Heidolph 2 rotary evaporator (Schwabach, Germany) to create a thin film in a 25

$170 \mathrm{~mL}$ round-bottom flask. The film was dried under vacuum for 1 hour to remove excess

171 solvent, during which the NP were washed with deionized (DI) water to remove excess

172 PVA. The dried film containing the NP was then rehydrated with $4 \mathrm{~mL}$ of DI water and

173 this solution was sonicated for 30 minutes, followed by freezing and lyophilization. After

174 lyophilization, the resulting NP were stored at $-20^{\circ} \mathrm{C}$. The ratio of lipid to NP during the

175 process was 1:5 (w/w). NP coated with DPPC and a small amount of DPPG NP were

176 synthesized using the same method with a 5:1 (w/w) DPPC:DPPG ratio and these NP are

177 referred to as DPPC-NP.

178

179 Nanoparticle Size and Surface Charge Characterization

180 Hydrodynamic diameter and surface charge of the NP were evaluated via dynamic

181 light scattering (DLS) and zeta potential analysis, respectively, using a Malvern Nano

182 Zetasizer (Malvern Instruments, Worcestershire, UK). The NP were diluted to $0.25 \mathrm{mg} / \mathrm{mL}$

183 in DI water and were analyzed at $25^{\circ} \mathrm{C}$ and an angle of $90^{\circ}$.

184

185 Evaluation of CUR NP Encapsulation Efficiency and Drug Loading

186 To determine the amount of CUR encapsulated in the NP systems, NP were 187 dissolved in DMSO $(1 \mathrm{mg} / \mathrm{mL})$ and the fluorescence of the solutions was analyzed using a 188 SpectraMax M2 Plate Reader (Molecular Devices, Sunnyville, CA) at an excitation of 420 
$189 \mathrm{~nm}$ and emission of $520 \mathrm{~nm}$. The encapsulation efficiency (EE) and drug loading were

190 calculated using the following equations:

191

192

$$
\text { Encapsulation Efficiency }(E E)=\frac{\text { experimental mass of drug in } N P}{\text { theoretical mass of drug in } N P} \times 100 \%
$$

$$
\text { Drug Loading }=\frac{\text { mass of drug in NP }}{\text { mass of } N P}
$$

196 Electron Microscopy Imaging Analysis of Nanoparticles

197 Images of the NP were taken using a Zeiss Sigma VP Field Emission-Scanning

198 Electron Microscope (FE-SEM) (Germany) for analysis of NP morphology. After NP were

199 suspended in basic water $(15 \mathrm{mg} / \mathrm{mL}), 1-2$ drops of this suspension were added to 200 aluminum SEM stubs (TedPella Inc., Redding, CA), and the samples were air dried. Dried 201 samples were then coated with a film of gold/palladium alloy using an Emscope SC400 202 sputter coating system at a $20 \mu \mathrm{A}$ for 75 seconds under argon. Images of the NP systems 203 were also captured via a JEOL JEM-2100F transmission electron microscope (TEM, 204 Peabody, MA) for observation of the NP core and outer layers. $1 \mu \mathrm{L}$ of $15 \mathrm{mg} / \mathrm{mL} \mathrm{NP}$ 205 suspension in water were placed on 200 square mesh copper grids (Election Microscopy 206 Sciences, Hatfield, PA) and air dried prior to imaging.

208 Differential Scanning Calorimetry (DSC) Analysis of Nanoparticles

209 Thermal phase transitions of the NP systems were analyzed using differential 210 scanning calorimetry (DSC) via a TA Q10 DSC system (TA Instruments, New Castle, DE) 
211 connected to an RSC-90 cooling accessory. For dry-state DSC, samples were lyophilized

21224 hours prior to analysis. $1-3 \mathrm{mg}$ of $\mathrm{NP}$ were analyzed at $10^{\circ} \mathrm{C} / \mathrm{min}$ from 0 to $300^{\circ} \mathrm{C}$. For

213 wet state analysis, NP were dispersed in DI water (0.1 mM DPPS) and $15 \mu \mathrm{L}$ of the NP

214 suspension was added to aluminum pans to be analyzed at $2{ }^{\circ} \mathrm{C} / \mathrm{min}$ and from 5 to $65^{\circ} \mathrm{C}$.

215 DSC analysis of the raw materials was also performed.

216

217 PVA Coating Quantification

218 Quantification of the amount of PVA on the surface of NP was completed using a 219 previously described method [45]. NP samples were dispersed in DI water (1 mg/mL). 400

$220 \mu \mathrm{L}$ of sample solution, $300 \mu \mathrm{L}$ of iodine solution $(1.25 \%$ iodine and $2.5 \%$ potassium

221 iodide), and $1.5 \mathrm{~mL}$ of $4 \%$ boric acid solution were mixed together for 20 minutes at room

222 temperature and $100 \mathrm{rpm}$. Absorbance of the samples was measured at a wavelength of $223630 \mathrm{~nm}$.

224

225 Phospholipid Content Quantification

226 Phospholipid quantification was carried out using Stewart's method [46]. Briefly,

$2272.7 \mathrm{~g}$ ferric chloride hexahydrate and $3 \mathrm{~g}$ of ammonium thiocyanate was dissolved in 100

$228 \mathrm{~mL}$ of distilled water. NP samples were dissolved in chloroform $(1 \mathrm{mg} / \mathrm{mL})$ and were

229 mixed with the ammonium ferrothiocyanate solution via vortexing for 5 minutes at a 2:1

$230(\mathrm{v} / \mathrm{v})$ ratio. After mixing, the solution was allowed to separate, and the phospholipid content

231 in the chloroform portion was measured via UV-vis spectroscopy at $488 \mathrm{~nm}$ (DPPC/G) and $232452 \mathrm{~nm}$ (DPPS).

233 
235 Release of CUR from the NP was carried out using a previously established 236 centrifugation technique [30]. $1 \mathrm{mg} / \mathrm{mL} \mathrm{NP}$ samples were dispersed in PBS (pH 7.4) or 237 sodium acetate buffer ( $\mathrm{pH} 5.35$ ) supplemented using $0.2 \%$ Tween 80 to enhance CUR 238 solubility. The suspensions were incubated at $37^{\circ} \mathrm{C}$ and $100 \mathrm{rpm}$ and at predetermined time 239 points the suspensions were removed and centrifuged at $23,102 \times g$ for 15 minutes. $200 \mu \mathrm{L}$ 240 of the supernatant was collected and frozen at $-20^{\circ} \mathrm{C}$ and $200 \mu \mathrm{L}$ of fresh medium was 241 added to the particle solution prior to re-dispersion and re-incubation. Release samples 242 were mixed with DMSO (1:1 by volume) prior to fluorescence spectroscopy analysis at an 243 excitation of $420 \mathrm{~nm}$ and emission of $520 \mathrm{~nm}$ [30].

\section{Cell Culture}

$246 \quad$ Murine macrophage cells (RAW 264.7) and A549 human adenocarcinoma cells

247 obtained from American Type Culture Collection (ATCC, Manassas, VA) were used for 248 cell culture studies. The cells were maintained at $37^{\circ} \mathrm{C}$ and $5 \% \mathrm{CO}_{2}$ in Dulbecco's modified 249 Eagle's medium (DMEM) supplemented with 10\% (v/v) fetal bovine serum, $100 \mathrm{U} / \mathrm{ml}$ 250 penicillin, $100 \mu \mathrm{g} / \mathrm{ml}$ streptomycin, Fungizone ${ }^{\circledR}(0.5 \mu \mathrm{g}$ amphotericin $\mathrm{B}, 0.41 \mu \mathrm{g} / \mathrm{mL}$ 251 sodium deoxycholate), and $1 \mathrm{mM}$ sodium pyruvate.

253 In Vitro Cytotoxicity Analysis of NP

254 The cytotoxic effect of the formulated NP on RAW 264.7 macrophages was 255 determined using a resazurin assay. Cells were seeded in 96 -well plate at 5,000 cells/well 256 and incubated overnight at $37^{\circ} \mathrm{C}$ and were then exposed to varying concentrations of PVA- 
257 CUR and DPPS-CUR NP (0.001 to $0.2 \mathrm{mg} / \mathrm{ml})$. Untreated cells were used as negative

258 controls. After 48 hours, resazurin solution $(60 \mu \mathrm{M})$ was added to the cells and incubated

259 for 3 hours. The fluorescence intensity of resorufin produced by viable cells was detected

260 at $544 \mathrm{~nm}$ (excitation) and $590 \mathrm{~nm}$ (emission) using BioTek Cytation 3 plate reader. The

261 relative viability of each sample was calculated by:

262

$$
\text { Relative Viability }=\frac{\text { Sample Fluorescence Intensity }}{\text { Control Fluorescence Intensity }} \times 100
$$

265 Nitrite Analysis

266 A Griess assay was performed to determine nitric oxide (NO) production by 267 macrophages. Following 48-hour incubation with varying concentrations of NP (CUR-

268 loaded and blank), 96-well plates containing the samples were centrifuged and $50 \mu \mathrm{L}$ of

269 the resulting supernatant was removed from each well. Griess reagents were added per 270 manufacturer's instructions and the absorbance was measured at $550 \mathrm{~nm}$. LPS (500 $271 \mathrm{ng} / \mathrm{mL}$ ), was used to promote NO production as a control.

273 In Vitro Cellular Uptake Via Spectroscopy and Confocal Microscopy

274 Cellular uptake of CUR-loaded NP by RAW 264.7 and A549 cells was observed 275 using a Cytation 3 image reader (BioTek, Winooski, VT). Cells (7,500 cells/well) were 276 seeded in a 96-well plate and incubated overnight at $37^{\circ} \mathrm{C}$. Cells were then incubated with

$2770.1 \mathrm{mg} / \mathrm{ml}$ of NP solutions and equal concentration of CUR for 1 and 3 hours. After 278 incubation, the media was removed, and the cells were washed three times with $200 \mathrm{mM}$ 279 glycine to remove any unbound NP that were not taken up by the cells. For quantification 
280 of cellular uptake, the fluorescence of the CUR within the cells was analyzed via 281 fluorescence spectroscopy at $420 \mathrm{~nm}$ (excitation) and $520 \mathrm{~nm}$ (emission).

282 RAW 264.7 and A549 cells were seeded into $35 \mathrm{~mm}$ glass-bottom petri dishes at a 283 concentration of 500,000 cells per dish and allowed to grow overnight. DPPS-CUR NP, 284 DPPC-CUR NP, or PVA-CUR NP were suspended and bath sonicated in cell culture 285 medium at $0.1 \mathrm{mg} / \mathrm{mL}$ and were then incubated with cells for 1 or 3 hours. Cells were 286 washed 3x in PBS and incubated in fresh media containing CellMask Deep Red 287 (Invitrogen) at $1 \mu \mathrm{L} / \mathrm{mL}$ media for 10 minutes. Cells were then fixed with $4 \%$ 288 paraformaldehyde for 10 minutes and rinsed 3x with PBS. Fresh PBS was added and cells 289 were immediately imaged using a Nikon Eclipse Ti2 inverted confocal fluorescent 290 microscope.

292 Statistical Analysis

293 All measurements were performed in at least triplicate. Statistical differences for in 294 vitro cellular studies was determined using one-way or two-way ANOVA with Tukey's 295 multiple comparisons test (GraphPad Prism version 7). For cumulative drug release, 296 Student's $t$-Test was used to determine statistical differences. A p-value of $<0.05$ or lower 297 was considered as statistically significant. Values are presented as mean \pm standard 298 deviation.

300 Results

301

302 Characterization of Ac-Dex Polymer and Nanoparticles 
Analysis of the acetal coverage and cyclic-to-acyclic (CAC) ratio of Ac-Dex

304 following its synthesis was confirmed via NMR. The acetal coverage of Ac-Dex was 73\%,

305 whereas the CAC ratio was $45 \%$. PVA-coated NP were synthesized using the emulsion

306 method to create NP with an Ac-Dex polymer core and PVA coating both with and without

307 curcumin loading (PVA-CUR NP and PVA-Blank NP, respectively). As seen in Table 1,

308 PVA-coated NP were 260-275 $\mathrm{nm}$ in diameter, exhibited polydispersity index (PDI) values

309 of 0.04 , and had nearly neutral surface charges (less than $-3 \mathrm{mV}$ ), as seen from the zeta

310 potential values.

311 After the synthesis of PVA-coated NP, the particles were coated with DPPS using

312 a thin film hydration method to form DPPS-coated NP, both with and without CUR loading

313 (DPPS-CUR NP and DPPS-Blank NP, respectively). The diameters for both the blank and

314 CUR-loaded DPPS NP increased by approximately $75 \mathrm{~nm}$ in comparison to the PVA-

315 coated NP. The PDI values were low for both DPPS NP systems (less than 0.21), however,

316 there was a substantial decrease in zeta potential, from $-3 \mathrm{mV}$ (PVA-coated NP) to $-40 \mathrm{mV}$

317 (DPPS-coated NP) for both blank and CUR-loaded NP systems.

318 The encapsulation efficiency (EE) and drug loading (by mass) of CUR-loaded NP

319 was analyzed to quantify the amount of CUR loaded in the particles. Both PVA- and DPPS-

320 coated NP exhibited EE values around $25 \%$ whereas the drug loading was 7.1 and $5.2 \mu \mathrm{g}$

321 CUR/mg NP for PVA-CUR NP and DPPS-CUR NP, respectively.

322

323 Morphological Analysis of NP Via Electron Microscopy

324 Scanning electron microscopy (SEM) and transmission electron microscopy (TEM)

325 were used to analyze the morphology and structure of PVA- and DPPS-coated NP loaded 
326 with CUR. SEM micrographs indicated that PVA-CUR NP presented spherical, smooth

327 morphology and were homogenous with respect to in size (Figure 1, top). TEM images

328 allowed for the visualization of a thin layer around the Ac-Dex polymer core, which likely

329 corresponds to the PVA coating. DPPS-CUR NP were fairly monodisperse with spherical,

330 smooth morphology as seen via SEM imaging (Figure 1, bottom). TEM micrographs

331 show a coating on the Ac-Dex NP core, likely indicating the DPPS coating on the NP.

\section{Coating Quantification}

334 The amount of PVA and total phospholipids present in the NP formulations were 335 quantified and are presented in Figure 2. There was significantly less PVA present on the

336 DPPS and DPPC-NP in comparison to the PVA-NP ( $p<0.001$ and $\mathrm{p}<0.0001$, 337 respectively). In addition, significantly more phospholipid was present in DPPS and 338 DPPC-NP in comparison to PVA-NP $(\mathrm{p}<0.01)$.

Differential Scanning Calorimetry (DSC) Thermal Analysis

341 Figure 3 shows the thermograms of the prepared NP and their corresponding raw

342 components in their dry states. An endothermic peak at $90^{\circ} \mathrm{C}$ was present, indicating a

343 bilayer phase transition for the DPPS-coated NP, which is slightly lower than the

344 endothermic phase transition temperature of raw DPPS at $99^{\circ} \mathrm{C}$. An endothermic peak was

345 present at $187^{\circ} \mathrm{C}$ for raw PVA, whereas a broadened endothermic peak was present at

$346195^{\circ} \mathrm{C}$ for PVA-coated NP. Both raw CUR and Ac-Dex exhibited endothermic peaks at

$347177^{\circ} \mathrm{C}$, signifying their melting points $[30,31,37]$. These prominent endothermic peaks

348 were not present in the formulated NP. The shift from $177^{\circ} \mathrm{C}$ to a broad peak at $195^{\circ} \mathrm{C}$ for 
349 PVA-coated NP could be due to the interaction between Ac-Dex and PVA. DPPS-coated

350 NP exhibited a broad endothermic peak at $165^{\circ} \mathrm{C}$, potentially due to the removal of the

351 PVA and the interaction between the Ac-Dex and DPPS. For wet-state DSC analysis of the

352 formulated NP, there were no measurable peaks present for the temperature range used;

353 curvature corresponded to the evaporation of water.

354

355 In Vitro Drug Release from NP

356 The release of CUR from the particles was analyzed at two $\mathrm{pH}$ values, including $357 \mathrm{pH} 7.4$ to simulate normal physiological $\mathrm{pH}$ and $\mathrm{pH} 5.35$ to approximate the $\mathrm{pH}$ in

358 macrophages [4]. Results in Figure 4 shows that there was no significant difference in the

359 cumulative release of CUR from PVA-coated versus DPPS-coated NP at pH 7.4 after 24

360 hours (5.2\% vs 6.3\%), signifying that the NP coating played no appreciable role in affecting

361 CUR release at this $\mathrm{pH}$. However, significantly more CUR was released at $\mathrm{pH} 5.35$ for

362 both DPPS- and PVA-coated particles (67.8\% and $88.8 \%$ release at $\mathrm{pH} 5.35$, respectively)

363 in comparison to $\mathrm{pH} 7.4$ ( $\mathrm{p}<0.001$ ), demonstrating the sensitivity of Ac-Dex to acidic

364 conditions. Furthermore, when comparing DPPS- and PVA-coated NP at $\mathrm{pH} 5.35$,

365 significantly more CUR was released from NP with a PVA coating, indicating the potential

366 influence of the DPPS layer in delaying the release of the cargo $(\mathrm{p}<0.05)$.

368 In Vitro Cytotoxicity Assay

369 The impact of the described NP systems on macrophage toxicity was evaluated and

370 the results are presented in Figure 5. These results indicate that the cells exhibited relative

371 viabilities similar to the control (media only) after 48 hours of exposure to the NP systems. 
372 Increasing the concentration of NP imparted no significant change in the viability of cells

373 for PVA-Blank NP, DPPS-Blank NP, or PVA-CUR NP for the concentrations tested.

374 However, for DPPS-CUR NP there was a slight but statistically significant decrease in the

375 viability of cells at $0.2 \mathrm{mg} / \mathrm{ml}$ in comparison to the control $(\mathrm{p}<0.05)$.

376

377 Nitrite Analysis

378 Following 48-hour incubation of NP samples on cells, CUR-loaded and blank

379 (control) NP groups produced significantly lower NO in comparison to LPS ( $\mathrm{p}<0.0001)$

380 (Figure 6). The NP groups were the same as the control group (media only) statistically,

381 indicating little to no production of NO [47].

382

383 In Vitro Cellular Uptake

384 The cellular uptake of CUR-loaded NP was evaluated in RAW 264.7 macrophages

385 and A549 adenocarcinoma cells for up to 3 hours as shown in Figure 6. Exploiting the

386 fluorescent properties of CUR, the presence of CUR-loaded NP within cells can easily be

387 detected via fluorescence imaging and spectroscopy. After 1 hour of exposure, significantly

388 more DPPS-coated NP were uptaken by macrophages and A549 cells in comparison to

389 PVA-coated NP, DPPC-coated NP, and raw CUR ( $<$ < 0.0001). For both cell types and all

390 formulations, uptake was not statistically different between 1 and 3-hour exposures. In

391 addition, significantly more NP were uptaken in RAW macrophages in comparison to

392 A549 cells for all NP formulations. Confocal microscopy confirmed fluorescence

393 spectroscopy quantification, showing higher fluorescence in macrophages in comparison

394 to A549 cells, indicating increased NP uptake. In addition, more NP were uptaken in DPPS- 
395 coated NP in comparison to DPPC- and PVA-coated systems in RAW and A549 cells.

396 Images indicate that CUR-loaded NP were primarily uptaken into the cytoplasm of

397 macrophages, especially for DPPS-CUR NP. On the other hand, there were minimal NP

398 located in the cytoplasm of A549 cells; instead the NP were more readily located in the

399 cellular membranes.

400

401 Discussion

402

403 Nanoparticle Design and Analysis

404 The use of Ac-Dex as a biodegradable NP core for drug delivery can allow for a 405 system with tunable and/or triggered degradation [32]. For the described NP systems, the 406 acetal coverage of Ac-Dex was similar to previous studies [30,31,37], whereas the CAC 407 ratio was slightly lower. The resulting Ac-Dex was favorable for the given application as

408 the adequate acetal coverage imparts hydrophobicity to Ac-Dex, allowing for NP 409 formulation and the lower $\mathrm{CAC}$ ratio will allow for faster drug release at acidic $\mathrm{pH}$ $410 \quad[30,31,35,37]$.

411 The increase in diameter and PDI from PVA-coated NP to DPPS-coated NP is

412 likely due to the presence of the DPPS coating around the Ac-Dex core. It has been shown

413 that an increase in NP diameter can lead to an increase in the uptake of NP into

414 macrophages, where particles $200 \mathrm{~nm}$ in diameter or greater are often subjected to a greater

415 amount of macrophage uptake [48-50]. For this study, larger NP were desired and

416 successfully formed (up to $350 \mathrm{~nm}$ for DPPS-coated NP), which was advantageous since

417 increased macrophage uptake was an objective [4]. While DPPS-coated NP exhibited 
418 higher PDI values then PVA-coated NP, these values were still low, indicating that all of

419 the formulated NP systems were homogenous with respect to size.

420 The surface charge of the NP, as indicated by zeta potential, decreased significantly

421 (from -3 to $-40 \mathrm{mV}$ ) upon coating with DPPS. The negative surface charge on the DPPS-

422 loaded NP is indicative of the polar head of DPPS being exposed to the outer environment,

423 similar to how apoptotic cells present DPPS to the outer leaflet of cells prior to engulfment

424 by macrophages $[6,28]$. The highly negatively surface charge is significantly lower than

425 the $-25 \mathrm{mV}$ threshold of being functionally negative, indicating that the NP are less likely

426 to aggregate due to electrostatic repulsion [51,52]. It should be noted that DPPC-coated NP

427 were also lower than the $-25 \mathrm{mV}$ threshold, indicating a functionally negative charge. In

428 addition, there were no differences in size, PDI value, or zeta potential upon the loading of

429 CUR on the NP core, indicating that the loading of the model drug had no significant effect

430 on the particle systems with respect to these characteristics.

431 CUR was encapsulated in the NP for two purposes: 1) to provide a model small

432 molecule that is easy to encapsulate owing to its hydrophobicity and 2) the inherent

433 fluorescence of CUR within the NP allows for easy fluorescence imaging and detection.

434 The encapsulation efficiency (EE) of CUR in the NP was lower than previously reported

435 values, which have ranged from 50-88\% [7,9,38]. However, since CUR was used as a

436 model small molecule, the EE values were satisfactory for this study. Upon coating the NP

437 with DPPS, CUR loading decreased slightly but the encapsulation efficiency stayed the

438 same, indicating that the DPPS coating did not significantly affect CUR loading.

439 The SEM and TEM micrographs indicate that the particles are spherical in shape

440 and monodisperse, which correlates to the PDI values for the systems. The DPPS-CUR NP 
441 seem to have slightly more coating on the NP surfaces, which could be due to the increased

442 presence of DPPS.

443

\section{Coating Quantification}

445 DPPS was selected to coat the nanoparticles owing to its ability to stimulate an

446 "eat-me" signal on apoptotic cells $[1,3,11,16,17]$. For cell studies, DPPC-NP were

447 produced as a control lipid system. Initially, the NP systems were coated with PVA because

448 it is one of the most widely used polymers in the pharmaceutical industry [45] and has been

449 extensively used as a NP coating agent due to the steric effects PVA coating imparts on

$450 \mathrm{NP}$, inhibiting aggregation. During the synthesis of phospholipid-coated NP, a significant

451 portion of the PVA was washed away prior to phospholipid coating, since there is

452 significantly less PVA present on phospholipid-coated NP in comparison to PVA NP

453 (Figure 2). It is also likely that the phospholipid coating shielded the remaining PVA upon

454 adsorption to the NP surface, as indicated by the low mass presence of PVA on

455 phospholipid-coated NP the high mass presence of total phospholipid on the NP, and the

456 changes in surface charge (Figure 2).

458 Thermal Analysis of Nanoparticle Formulations

459 Dry-state DSC thermograms indicated the presence of DPPS in the DPPS-coated

$460 \mathrm{NP}$ via the bilayer phase transition peak that occurred at $90^{\circ} \mathrm{C}$ for the NP, which is slightly

461 lower than the raw DPPS endothermic peak present at $99^{\circ} \mathrm{C}$. This transition signifies the

462 presence of bilayer formation of the phospholipid around the Ac-Dex polymer core in its

463 dry state. The decrease in the transition temperature is likely due to the interaction of the 
464 multiple components in the formulation. As the transition temperatures of the NP

465 formulations were just below or well above $100^{\circ} \mathrm{C}$, they are likely to be stable in the dry

466 state and can be easily reconstituted before use [53]. The endothermic peak that raw CUR

467 exhibited at $177^{\circ} \mathrm{C}$ was not observed in CUR-loaded NP. According to our previous

468 research (data not shown), this is likely due a limitation in DSC detection with relation to

469 the actual amount of CUR in the samples.

470 In studies using liposomal formulations, DSC is used to assess the transition

471 temperature(s) in the systems in order to determine the presence and state of bilayers and

472 the differences associated between blank liposomes and modified/drug-loaded systems

$473[43,44]$. For wet-state analysis (Figure 3C), there was no endothermic peak present around

$47450-60^{\circ} \mathrm{C}$, which is the temperature at which the gel/crystalline phase transition occurs for

475 PS bilayers in aqueous solutions [24,54]. Such a phenomenon could have occurred based

476 on the preparation method used, as described previously. In certain cases, self-assembly of

477 polymer-lipid structures can occur via hydrophobic interaction between the lipid tails and

478 a polymeric core simultaneously with hydrophilic interactions between the polar head and

479 external environment [55]. Due to the concentration dependency of DSC, it is more likely

480 that the DPPS concentration may have been too low to be detectable by thermal analysis,

481 like that of the CUR detection limit mentioned previously. It should be noted that the

482 curvature of the thermograms in Figure 3C corresponds to the evaporation of water, which

483 is not shown in the thermogram. Overall, the results indicate the presence of DPPS on the

484 surface of the NP, further strengthening what has been previously described with respect

485 to successful DPPS coating of the described systems. 
488 The release from CUR from PVA- and DPPS-coated NP was evaluated to 489 demonstrate the ability of the system to provide sustained release of a therapeutic agent 490 and to show the acid-sensitive nature of Ac-Dex. Ac-Dex is known to degrade more quickly

491 in acidic environments [32]. In a previous study, Ac-Dex particles released $60 \%$ of their

492 camptothecin payload after 7 days [35] and 15\% of rapamycin after 10 days [4] at $\mathrm{pH}$ of

493 7.4. Comparatively, at $\mathrm{pH}$ values of 5 or lower, Ac-Dex particles released $100 \%$ of their

494 content in as little as 24 hours [4,30,33]. For this study, at pH 5.35, 9.3\% of CUR was

495 initially released from DPPS-CUR NP and 29.2\% was released after 1 hour, followed by a

496 sustained release of cargo of $67.8 \%$ after 24 hours, when equilibrium is reached. At pH 7.4,

497 both NP systems exhibited minimal CUR release, demonstrating the stability of Ac-Dex in

498 neutral $\mathrm{pH}$. However, both systems exhibited triggered release at $\mathrm{pH} 5.35$, exhibiting a

499 significantly higher total release in comparison to their $\mathrm{pH} 7.4$ counterparts $(\mathrm{p}<0.001)$.

500 Overall, these data indicate a more stable NP formulation at $\mathrm{pH} 7.4$ and the potential for

501 triggered release at $\mathrm{pH} 5.35$, corresponding to stability in circulation and burst release in

502 macrophages, respectively.

503

504 Cytotoxic Analysis and Macrophage Uptake of Nanoparticles

505 To assess the in vitro cytotoxicity of the NP formulations on RAW 264.7

506 macrophages, the cells were exposed to the systems for 48 hours. It was observed that the

507 NP systems were not toxic to the cells, however, a slight, but significant, decrease in

508 viability of RAW 264.7 cells for DPPS-CUR NP at $0.2 \mathrm{mg} / \mathrm{ml}$ was observed (Figure 5).

509 This decrease in viability was minimal and could easily be overcome by using lower 
510 concentrations of the particles in future studies. Overall, these results indicate the safety of

511 the described NP for a time period longer than necessary for NP uptake into macrophages,

512 indicating that they are safe to use in the intended fashion.

513 Nanomaterials may provoke immune and inflammation responses following

514 interactions with multiple biological entities [49]. To assess inflammatory and immune

515 response stimulation, NO production was measured using a Griess assay, where LPS was

516 used as a positive control to stimulate NO production in RAW macrophages. It was

517 observed that NP at varying concentrations stimulated significantly less NO production in

518 comparison to LPS and resulted in NO concentrations comparable to that of the control

519 (media only). Results indicated that blank NP caused NO production at concentrations

520 similar to that of the CUR-loaded NP. These data indicate both blank and CUR-loaded

521 formulations produced minimal NO and that CUR did not play an active role in the

522 inhibition of NO production.

523 Since the purpose of this study was to formulate acid-sensitive NP capable of

524 enhanced macrophage uptake, the ability of DPPS-coated NP to be uptaken by

525 macrophages was evaluated, with A549 cells acting as control tissue. PVA and DPPC-

526 coated NP were used as other coatings for comparison to DPPS. Several environmental

527 and physicochemical factors are known to influence the cellular uptake of NP into

528 macrophages. The preferential uptake of DPPS-CUR NP by macrophages in comparison

529 to PVA-CUR NP and DPPC-CUR NP is likely due to the DPPS coating on the Ac-Dex

530 polymeric core. Confocal microscopy in tandem with fluorescence quantification of CUR-

531 loaded NP within the cells confirm the uptake of DPPS-CUR-NP and localization to the

532 cytoplasm of macrophages. The presence of DPPS on the cellular surface is known to cause 
533 an "eat-me" signal in cells that are transitioning toward an apoptotic state, causing

534 macrophages to identify and phagocytose the dying cells $[1-3,7,11]$. Therefore, in terms of

535 recognition, the DPPS coating on the Ac-Dex NP was identified by the macrophages,

536 which produced the necessary phagocytotic action, resulting in cellular uptake of the

537 particles $[1,6,7,16,25,27]$. In most cases, apoptosis is initiated by the presence DPPS and

538 other signaling pathways such as receptor-ligand interactions or identification of PS

539 binding proteins produced by phagocytes. However, there is a debate as to whether DPPS

540 recognition by itself is sufficient for the uptake of apoptotic cells by phagocytotic cells

$541[3,25]$. In the case of nanoparticles, is has been shown that a DPPS coating alone is

542 sufficient for uptake, as seen in this current study and otherwise $[7,16,17]$.

543 Another consideration for cellular uptake of the described particles is in terms of

544 their physicochemical characteristics. Stimulation of phagocytosis of nanoparticle-based

545 delivery systems via macrophages is also dependent on the size and shape of the particles.

546 Since the DPPS-CUR NP were greater than $300 \mathrm{~nm}$ in diameter, it is likely that these NP

547 were phagocytosed not only because of the DPPS layer, but also because of their size

$548[4,48,56]$. It is well known that surface charge can play a role in biological interactions.

549 Interestingly, surface charge did not play a role in the uptake of the NP, as shown by the

550 similar charges of DPPC and DPPS-coated NP that produced significantly different uptake

551 quantities $[48,51,52]$. This phenomenon could also explain the minimal phagocytosis of

552 the PVA-coated NP and confirm the effect of DPPS on the uptake of the NP. Overall, the

553 results indicate the successful uptake of DPPS-coated NP into macrophages, indicating that

554 these systems could be used for the treatment of a multitude of diseases involving infected

555 cells. 


\section{Conclusions}

558 Macrophages can harbor infectious agents that lead to potentially fatal diseases

559 such as TB and HIV. Due to this, treatment of these diseases is challenging and therefore,

560 the goal of this study was to design a delivery system that would enhance the uptake of

561 nanoparticles into macrophages, allowing for targeted delivery, a decrease of systemic side

562 effects, and decreased treatment times. Results showed that both PVA-coated and DPPS-

563 coated NP were monodisperse and that there was a significant increase in size upon DPPS

564 coating. The surface charge of the DPPS NP was $-40 \mathrm{mV}$, indicating that DPPS was present

565 on the surface of the NP. This was further confirmed via PVA quantification, which

566 indicated minimal amounts of PVA on the DPPS NP. DSC results confirmed the presence

567 of a DPPS bilayer on the NP surface and the likely stability of the NP during synthesis and

568 storage conditions. CUR-loaded NP successfully encapsulated CUR and this agent was

569 more quickly released in acidic conditions in comparison to neutral $\mathrm{pH}$ owing to the acid

570 sensitivity of Ac-Dex. In addition, CUR-loaded NP exhibited sustained release of CUR.

571 PVA-coated NP were not readily phagocytosed by macrophages, whereas DPPS-coated

572 NP were phagocytosed within 1 hour of exposure, showing the physiological relevance of

573 the DPPS coating for the enhanced delivery to macrophages. These data show that the

574 particles can potentially deliver therapeutic agents throughout the body with fewer

575 concerns of systemic drug exposure, and when exposed to macrophages, the particles are

576 capable of being phagocytosed relatively quickly. Therefore, the combination of Ac-Dex

577 and DPPS can be a viable option for targeted delivery for macrophage-associated diseases. 


\section{Conflicts of Interest}

580 The authors have no conflicts of interest to report for this study.

\section{Acknowledgements}

583 The authors thank Dr. Richard Kingsley for assistance with TEM imaging, Mitch

584 Gravely for confocal imaging, and $\mathrm{RIN}^{2}$ for SEM, TEM, and DSC access. Research

585 reported in this publication was partially supported by the Institutional Development

586 Award (IDeA) Network for Biomedical Research Excellence from the National Institute

587 of General Medical Sciences of the National Institutes of Health under grant number

588 P20GM103430. In addition, this work was made possible by the use of equipment and

589 services available through the RI-INBRE Centralized Research Core Facility. This material

590 is based upon work conducted at a Rhode Island NSF EPSCoR research facility, supported

591 in part by the National Science Foundation EPSCoR Cooperative Agreement \#EPS-

592 1004057. This material is also based in part upon work supported by the National Science

593 Foundation under grant \#1508868. Any opinions, findings, and conclusions or

594 recommendations expressed in this material are those of the authors and do not necessarily

595 reflect the view of the National Science Foundation or National Institutes of Health.

596

597

598

599 Tables and Figures

600 
601 Table 1. Diameter, polydispersity index (PDI), surface charge via zeta potential,

602 curcumin encapsulation efficiency, and drug loading of nanoparticle systems.

\begin{tabular}{|c|c|c|c|c|c|}
\hline & $\begin{array}{c}\text { Diameter } \\
(\mathbf{n m})\end{array}$ & PDI & $\begin{array}{c}\text { Zeta } \\
\text { Potential } \\
(\mathbf{m V})\end{array}$ & $\begin{array}{c}\text { Drug } \\
\text { Efficiency }(\%)\end{array}$ & $\begin{array}{c}\text { Druading }(\boldsymbol{\mu g} \\
\mathbf{d r u g} / \mathbf{m g} \\
\mathbf{p a r t i c l e})\end{array}$ \\
\hline PVA-Blank NP & $262.6 \pm 1.9$ & $0.04 \pm 0.00$ & $-2.2 \pm 3.5$ & --- & --- \\
\hline PVA-CUR NP & $272.6 \pm 10.4$ & $0.04 \pm 0.01$ & $-3.0 \pm 0.6$ & $25.1 \pm 0.1$ & $7.1 \pm 1.8$ \\
\hline DPPS-Blank NP & $335.6 \pm 3.8$ & $0.21 \pm 0.43$ & $-40.4 \pm 3.3$ & --- & --- \\
\hline DPPS-CUR NP & $350.5 \pm 16.9$ & $0.13 \pm 0.65$ & $-40.6 \pm 1.3$ & $24.5 \pm 0.1$ & $5.2 \pm 0.7$ \\
\hline
\end{tabular}

603

604

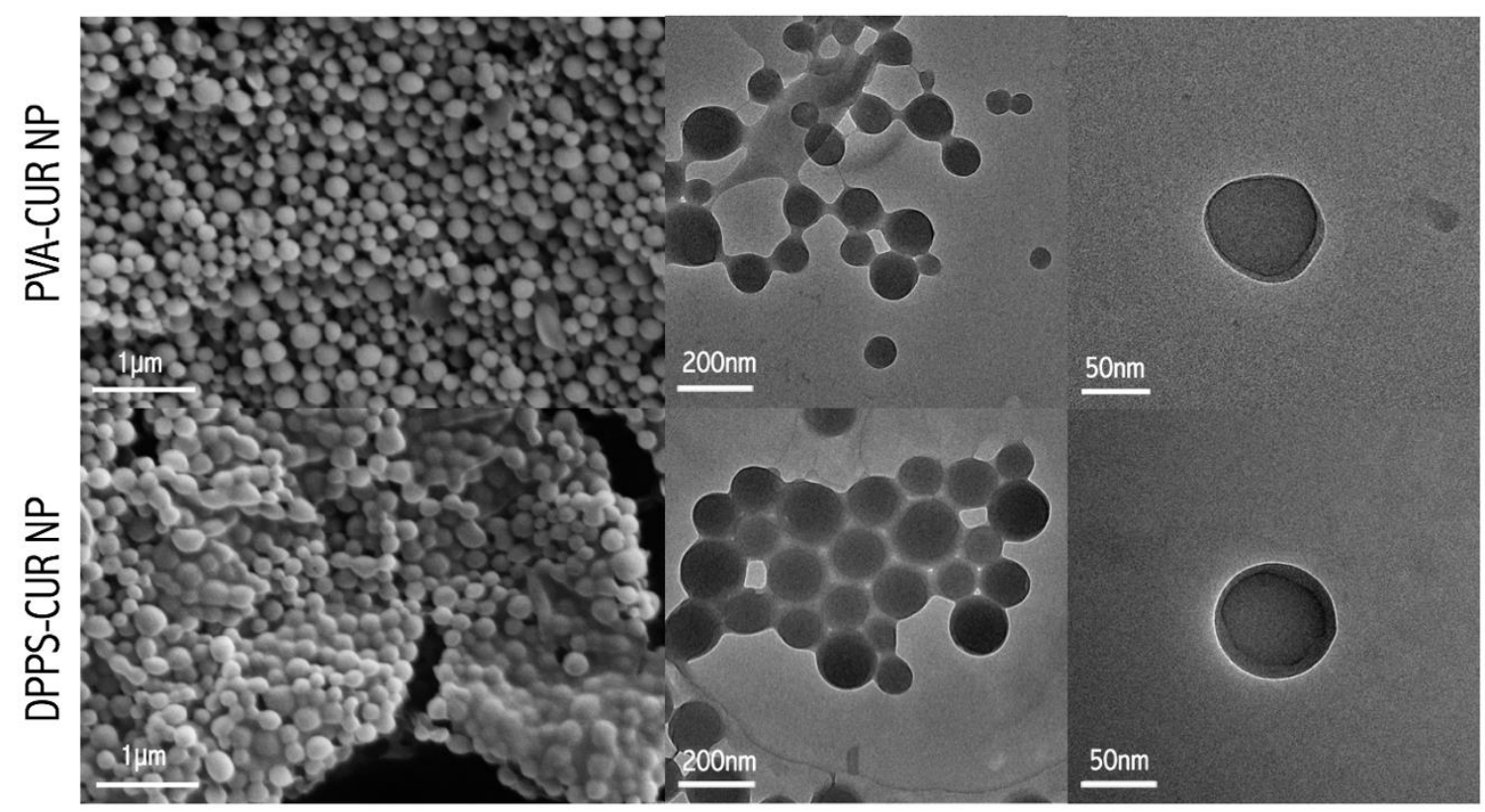

605 Figure 1. Representative SEM (left) and TEM (middle, right) micrographs of PVA- and

606 DPPS-coated, CUR-loaded nanoparticles (PVA-CUR NP and PVA-DPPS NP,

607 respectively).

608 


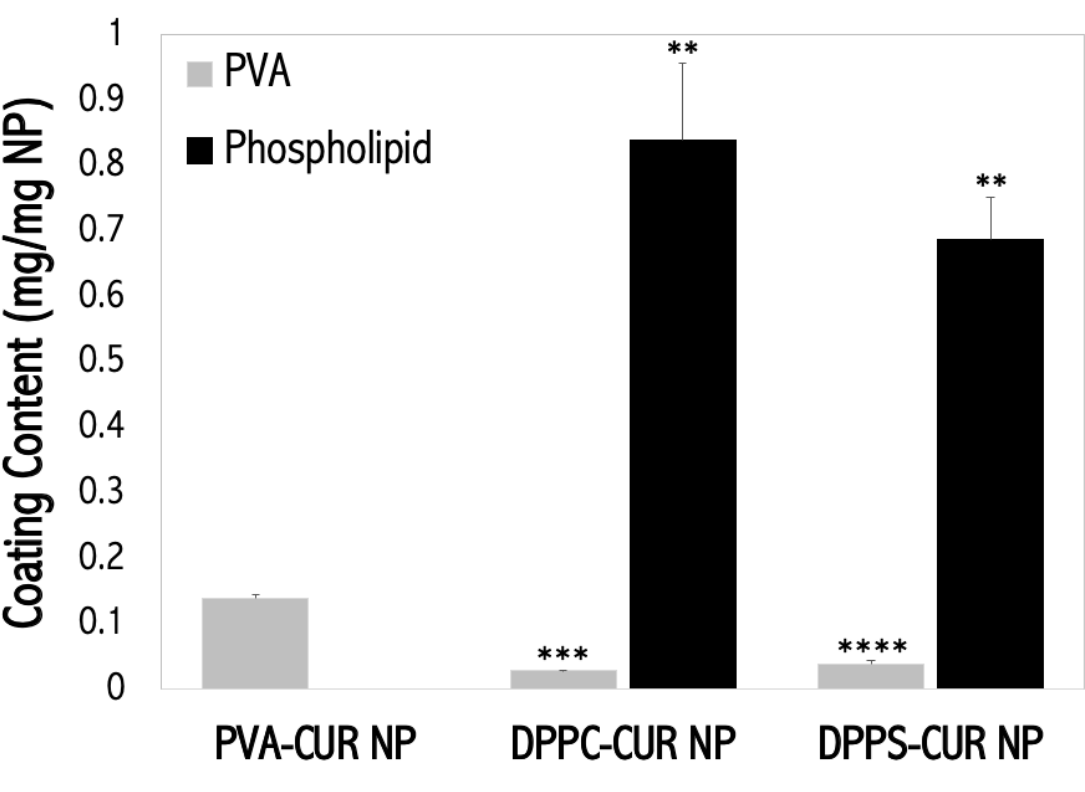

610 Figure 2. Quantification of the amount of poly(vinyl alcohol) (PVA) and total

611 phospholipid in PVA-CUR, DPPC-CUR, and DPPS-CUR nanoparticles (NP) $(* \mathrm{p}<0.05$,

$612 * * \mathrm{p}<0.01, * * * \mathrm{p}<0.001$ in comparison to PVA-CUR NP). 

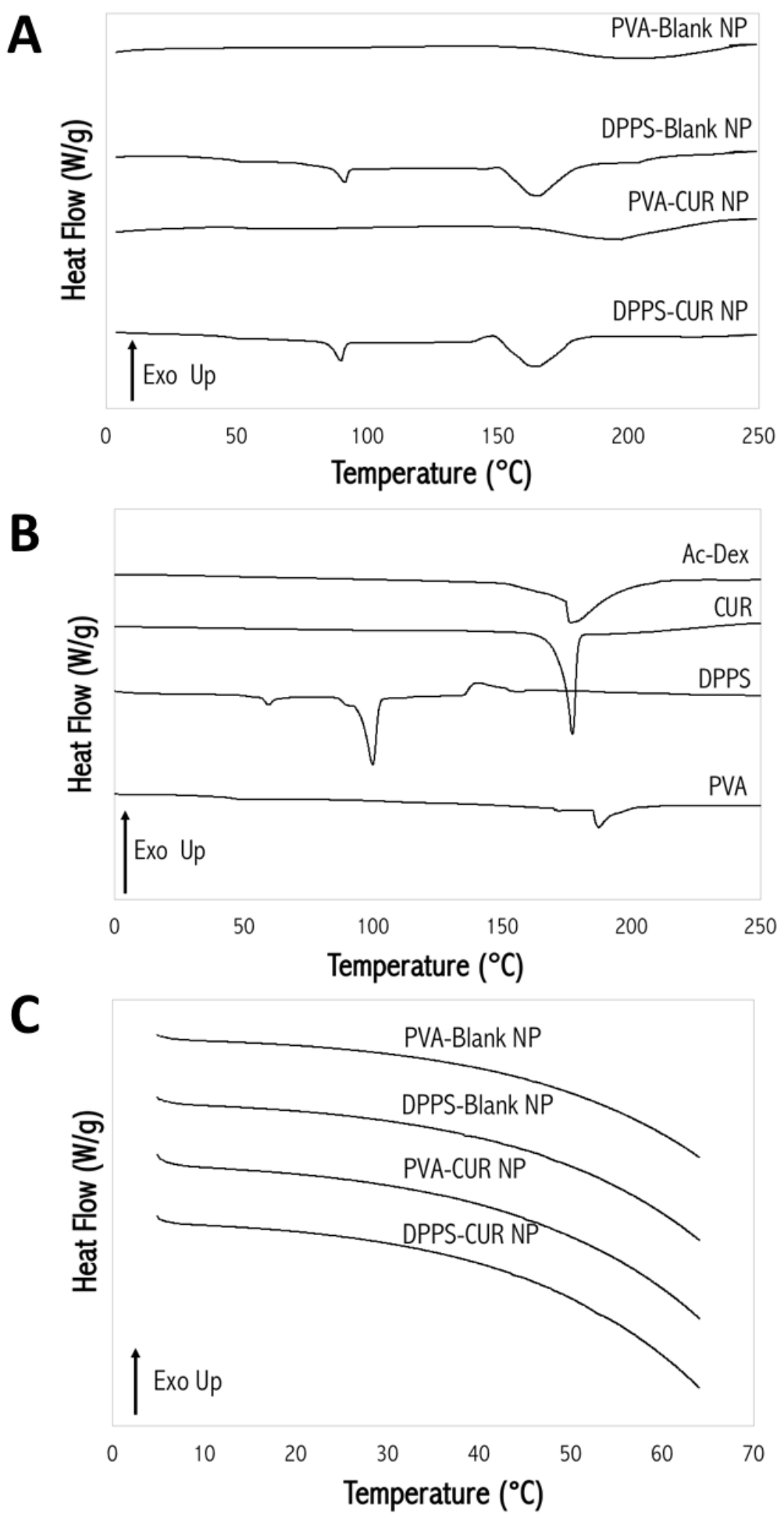

614 Figure 3. Differential scanning calorimetry (DSC) thermograms of: (A) formulated

615 nanoparticles and (B) the raw components that make up the nanoparticle systems in their

616 dry state, and (C) formulated nanoparticles in their wet state. 


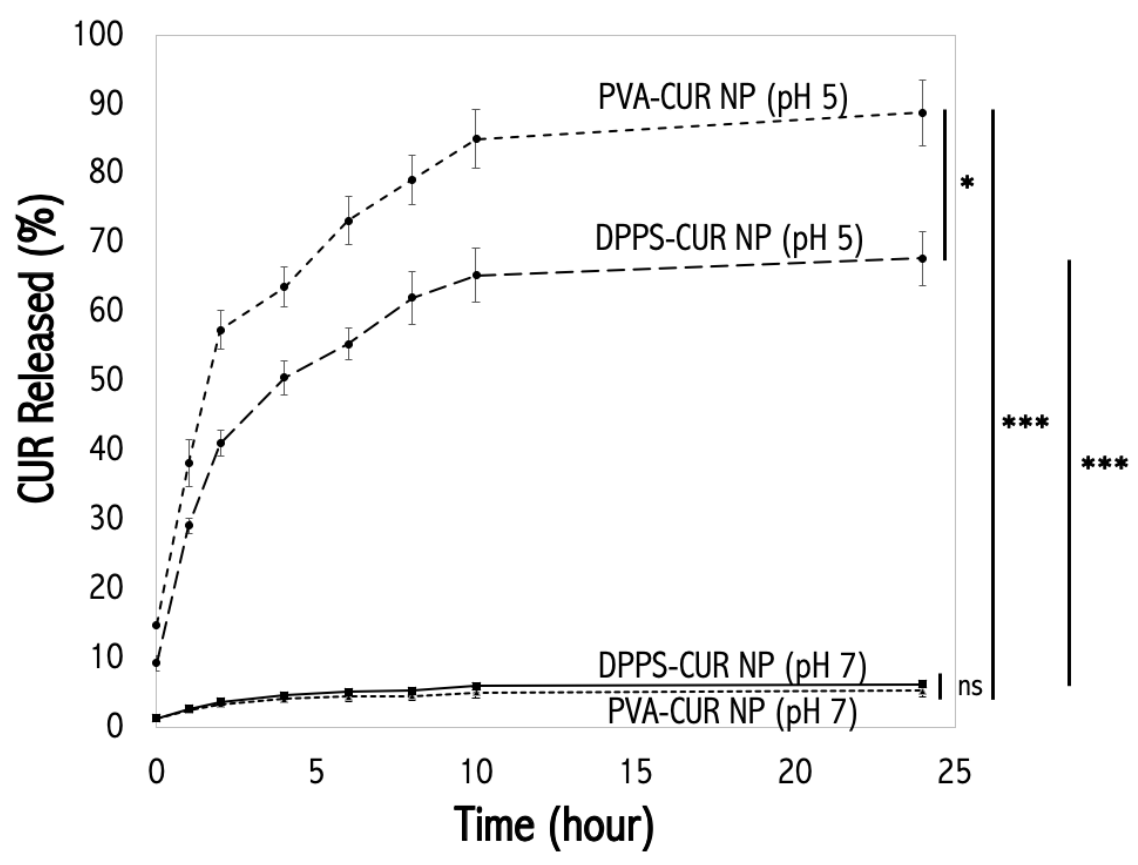

618 Figure 4. Cumulative release of curcumin (CUR) from nanoparticle systems at $\mathrm{pH} 7$ and $619 \mathrm{pH} 5(* \mathrm{p}<0.05, * * \mathrm{p}<0.01, * * * \mathrm{p}<0.001, \mathrm{~ns}=$ non-significant $)$.

620

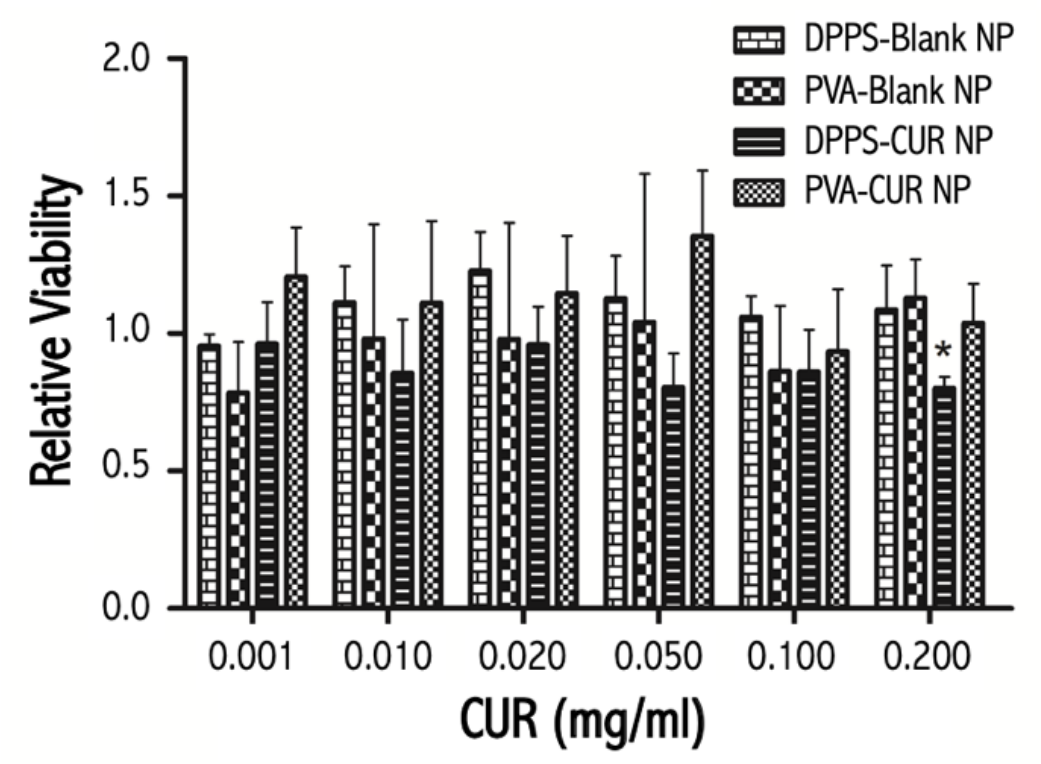

621

622 Figure 5. Cytotoxicity analysis (relative viability) of nanoparticle systems on RAW

623264.7 macrophages after 48 hours of exposure compared to control ( $* \mathrm{p}<0.05)$. 


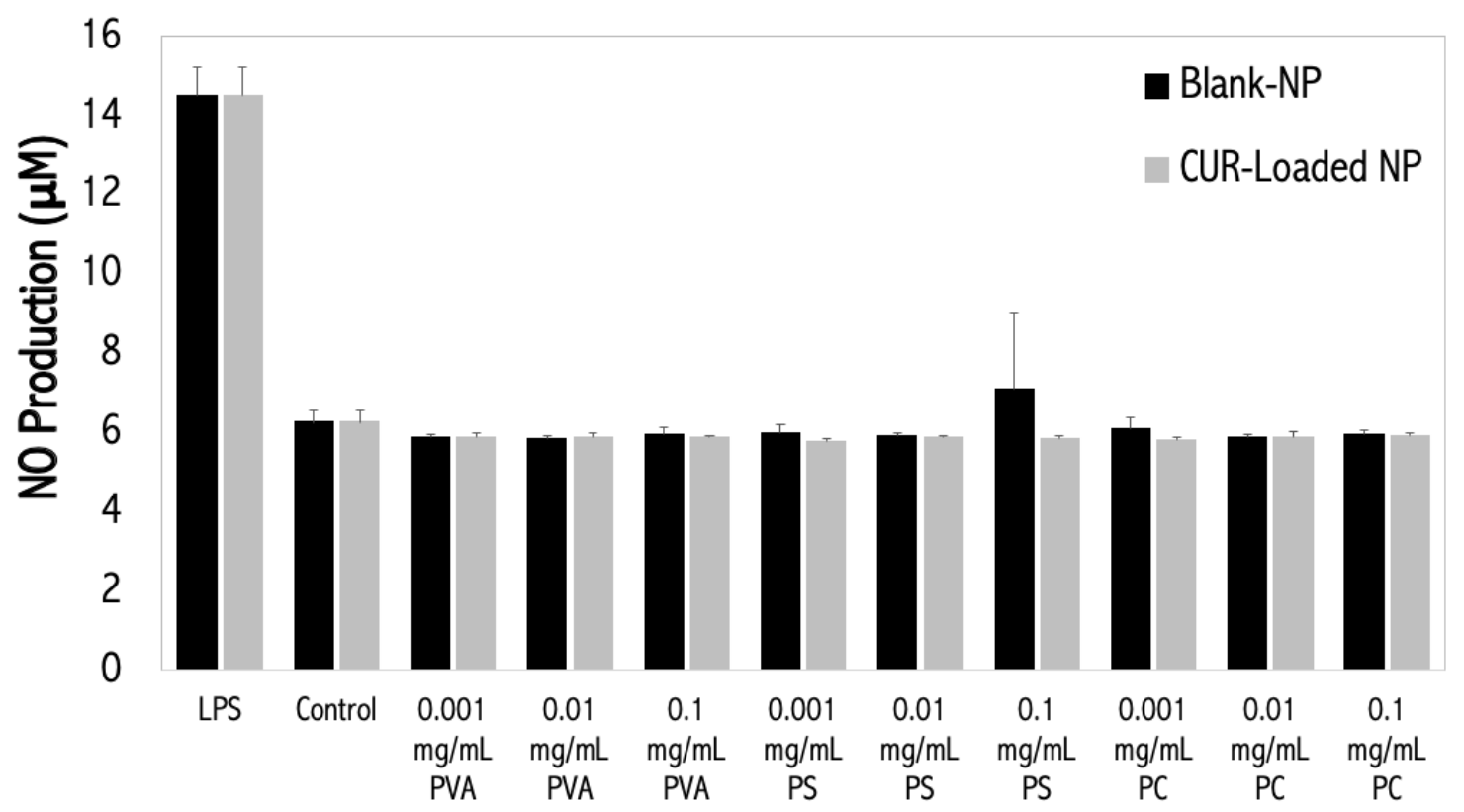

625 Figure 6. Nitric oxide (NO) production from RAW 264.7 macrophages incubated for 48

626 hours with varying concentrations of nanoparticle formulations or LPS. No statistical

627 significance was observed between the groups and control group (no treatment) and all

628 were statistically lower than the LPS sample $(\mathrm{p}<0.0001)$.

629

630

631

632

633 

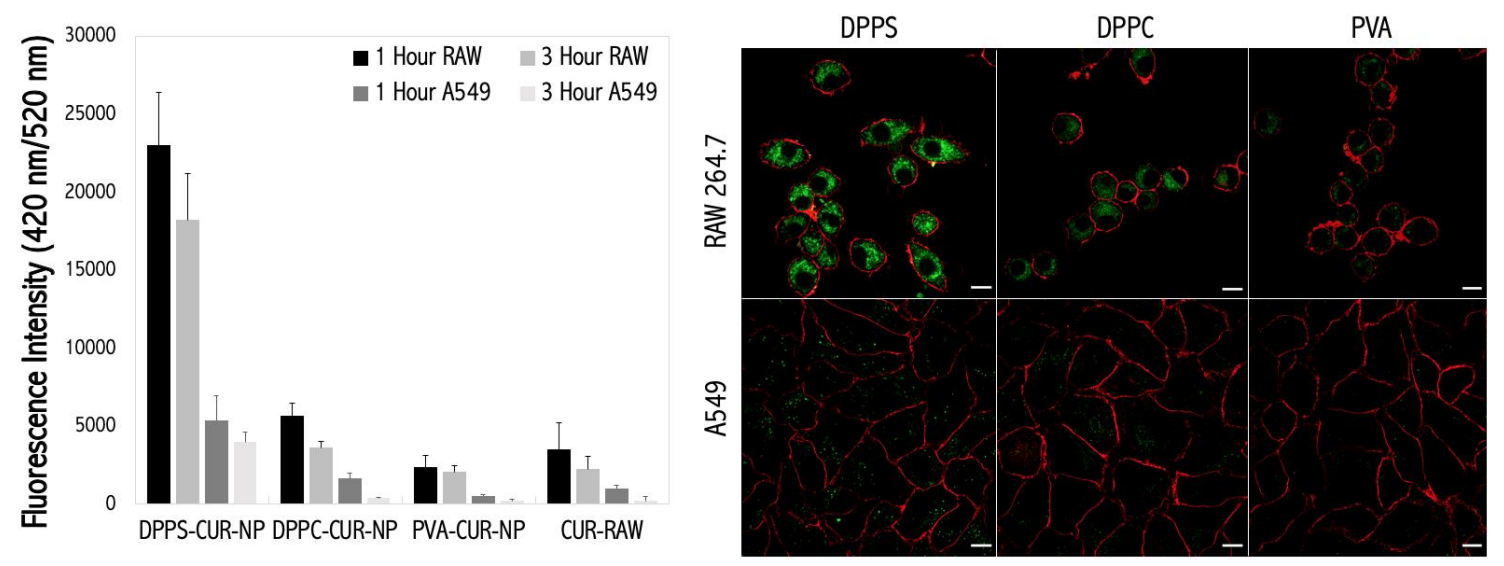

635 Figure 7. (Left) Fluorescence spectroscopy analysis of nanoparticle uptake into cells and

636 (Right) representative confocal images of nanoparticle uptake by RAW 264.7 macrophages

637 and A549 cells (scale bar $=10 \mu \mathrm{m})$.

638

639

640

641

642

643

644

645

646

647

648

649

650

651 


\section{References}

654 [1] V. Bagalkot, J.A. Deiuliis, S. Rajagopalan, A. Maiseyeu, "Eat me" imaging and 655 therapy, Adv. Drug Deliv. Rev. 99 (2016) 2-11. doi:10.1016/j.addr.2016.01.009.

656 [2] F. Ahsan, I.P. Rivas, M.A. Khan, A.I. Torres Suárez, Targeting to macrophages:

657 Role of physicochemical properties of particulate carriers - Liposomes and 658 microspheres - On the phagocytosis by macrophages, J. Control. Release. 79 659 (2002) 29-40. doi:10.1016/S0168-3659(01)00549-1.

660 [3] K.S. Ravichandran, Beginnings of a Good Apoptotic Meal: The Find-Me and Eat661 Me Signaling Pathways, Immunity. 35 (2011) 445-455.

662 doi:10.1016/j.immuni.2011.09.004.

663 [4] K.J. Kauffman, N. Kanthamneni, S.A. Meenach, B.C. Pierson, E.M. Bachelder, 664 K.M. Ainslie, Optimization of rapamycin-loaded acetalated dextran microparticles 665 for immunosuppression, Int. J. Pharm. 422 (2012) 356-363.

666 doi:10.1016/j.ijpharm.2011.10.034.

667 [5] A. Matsumoto, Y. Takahashi, M. Nishikawa, K. Sano, M. Morishita, C.

668 Charoenviriyakul, H. Saji, Y. Takakura, Role of Phosphatidylserine-Derived

669 Negative Surface Charges in the Recognition and Uptake of Intravenously Injected 670 B16BL6-Derived Exosomes by Macrophages, J. Pharm. Sci. 106 (2016) 168-175. 671 doi:10.1016/j.xphs.2016.07.022.

672 [6] R.A. Petazzi, A. Gramatica, A. Herrmann, S. Chiantia, Time-controlled 673 phagocytosis of asymmetric liposomes: Application to phosphatidylserine 674 immunoliposomes binding HIV-1 virus-like particles, Nanomedicine 
$675 \quad$ Nanotechnology, Biol. Med. 11 (2015) 1985-1992.

676 doi:10.1016/j.nano.2015.06.004.

677 [7] J. Wang, Y.-X. Kang, W. Pan, W. Lei, B. Feng, X.-J. Wang, Enhancement of Anti678 Inflammatory Activity of Curcumin Using Phosphatidylserine-Containing

$679 \quad$ Nanoparticles in Cultured Macrophages, Int. J. Mol. Sci. 17 (2016) 969. 680 doi:10.3390/ijms17060969.

681 [8] L. Zhang, H. Zhou, O. Belzile, P. Thorpe, D. Zhao, Phosphatidylserine-targeted 682 bimodal liposomal nanoparticles for in vivo imaging of breast cancer in mice, J. 683 Control. Release. 183 (2014) 114-123. doi:10.1016/j.jconrel.2014.03.043.

684 [9] Y. Zheng, Y. Chen, L. Jin, H. Ye, G. Liu, Cytotoxicity and Genotoxicity in Human 685 Embryonic Kidney Cells Exposed to Surface Modify Chitosan Nanoparticles 686 Loaded with Curcumin, AAPS PharmSciTech. (2015). doi:10.1208/s12249-015$687 \quad 0471-1$

688 [10] J. Van Ingen, R.E. Aarnoutse, P.R. Donald, A.H. Diacon, R. Dawson, G. Plemper 689 Van Balen, S.H. Gillespie, M.J. Boeree, Why do we use $600 \mathrm{mg}$ of rifampicin in 690 tuberculosis treatment?, Clin. Infect. Dis. 52 (2011). doi:10.1093/cid/cir184.

691 [11] V. Bagalkot, M.A. Badgeley, T. Kampfrath, J.A. Deiuliis, S. Rajagopalan, A. 692 Maiseyeu, Hybrid nanoparticles improve targeting to inflammatory macrophages 693 through phagocytic signals, J. Control. Release. 217 (2015) 243-255. 694 doi:10.1016/j.jconrel.2015.09.027.

695 [12] S.H.M. Chachuli, A. Nawaz, K. Shah, I. Naharudin, T.W. Wong, In Vitro 696 Investigation of Influences of Chitosan Nanoparticles on Fluorescein Permeation 697 into Alveolar Macrophages, Pharm. Res. (2016) 1-12. doi:10.1007/s11095-016- 
$1893-5$.

699 [13] I.M. El-sherbiny, H.D.C. Smyth, Controlled Release Pulmonary Administration of $700 \quad$ Curcumin Using Swellable Biocompatible Microparticles, Mol Pharm. (2012).

701 [14] V.A. Fadok, D.R. Voelker, P.A. Campbell, J.J. Cohen, D.L. Bratton, P.M. Henson, 702

[16] T. Geelen, S.Y. Yeo, L.E.M. Paulis, L.W.E. Starmans, K. Nicolay, G.J. Strijkers, [15] D.P. Gaspar, V. Faria, L.M.D. Gonçalves, P. Taboada, C. Remuñán-López, A.J. Almeida, Rifabutin-loaded solid lipid nanoparticles for inhaled antitubercular therapy: Physicochemical and in vitro studies, Int. J. Pharm. 497 (2016) 199-209. doi:10.1016/j.ijpharm.2015.11.050.

[18] K.R. Peterson, M.A. Cottam, A.J. Kennedy, A.H. Hasty, Macrophage-Targeted Therapeutics for Metabolic Disease, Cell Press. 39 (2018) 536-546. doi:10.1016/j.tips.2018.03.001.

[19] A. Costa, B. Sarmento, V. Seabra, Mannose-functionalized solid lipid nanoparticles are e ff ective in targeting alveolar macrophages, Eur. J. Pharm. Sci. 
114 (2018) 103-113. doi:10.1016/j.ejps.2017.12.006.

722 [20] E. Maretti, L. Costantino, C. Rustichelli, E. Leo, M. Antonietta, F. Buttini, E.

723 Truzzi, V. Iannuccelli, Surface engineering of Solid Lipid Nanoparticle assemblies

724 by methyl a - D -mannopyranoside for the active targeting to macrophages in anti-

725 tuberculosis inhalation therapy, Int. J. Pharm. 528 (2017) 440-451.

726 doi:10.1016/j.ijpharm.2017.06.045.

727 [21] L. Zou, Y. Tao, G. Payne, L. Do, T. Thomas, J. Rodriguez, H. Dou, Targeted

728 delivery of nano-PTX to the brain tumor-associated macrophages, Oncotarget.

$729 \quad$ (2016) $1-15$.

730 [22] K. Binnemars-postma, G. Storm, J. Prakash, Nanomedicine Strategies to Target

731 Tumor-Associated Macrophages, Int. J. Mol. Sci. 18 (2017) 1-27.

732 doi:10.3390/ijms18050979.

733 [23] N. Mubin, M.S. Umar, S. Zubair, M. Owais, Selective Targeting of Functionalized

734 Mycobacterium tuberculosis Protein Loaded Chitosan Nanoparticle to

735 Macrophages : Correlation With Activation of Immune System, Front. Microbiol.

7369 (2018) 1-15. doi:10.3389/fmicb.2018.02469.

737 [24] R.N. Lewis, R.N. McElhaney, Calorimetric and spectroscopic studies of the

738 thermotropic phase behavior of lipid bilayer model membranes composed of a

739 homologous series of linear saturated phosphatidylserines., Biophys. J. 79 (2000)

$740 \quad$ 2043-2055. doi:10.1016/S0006-3495(00)76452-6.

741 [25] K. Segawa, S. Nagata, An Apoptotic "Eat Me" Signal: Phosphatidylserine

742 Exposure, Trends Cell Biol. 25 (2015) 639-650. doi:10.1016/j.tcb.2015.08.003.

743 [26] F. Badalà, K. Nouri-mahdavi, D.A. Raoof, Apoptotic cell clearance: basic biology 
and therapeutic potential, Nat Rev Immunol. 144 (2008) 724-732. doi:10.1038/jid.2014.371.

746 [27] M. Manrique-moreno, L. Heinbockel, M. Suwalsky, P. Garidel, K. Brandenburg, Biochimica et Biophysica Acta Biophysical study of the non-steroidal anti-in fl ammatory drugs ( NSAID ) ibuprofen , naproxen and diclofenac with phosphatidylserine bilayer membranes, BBA - Biomembr. 1858 (2016) 21232131. doi:10.1016/j.bbamem.2016.06.009. nanovesicles: Synergism with paclitaxel in human lung adenocarcinoma A549 cells, J. Control. Release. 156 (2011) 413-420. doi:10.1016/j.jconrel.2011.07.025.

754 [29] H. Hosseini, Y. Li, P. Kanellakis, C. Tay, A. Cao, P. Tipping, A. Bobik, B.H. Toh, 755 T. Kyaw, Phosphatidylserine liposomes mimic apoptotic cells to attenuate

[30] Z. Wang, S.K. Gupta, S.A. Meenach, Development and physicochemical atherosclerosis by expanding polyreactive IgM producing B1a lymphocytes, Cardiovasc. Res. 106 (2015) 443-452. doi:10.1093/cvr/cvv037.

765

[32] E.M. Bachelder, E.N. Pino, K.M. Ainslie, Acetalated Dextran: A Tunable and 
Chem. Rev. 117 (2017) 1915-1926. doi:10.1021/acs.chemrev.6b00532.

768 [33] K.E. Broaders, J. a Cohen, T.T. Beaudette, E.M. Bachelder, J.M.J. Fréchet,

769 Acetalated dextran is a chemically and biologically tunable material for particulate

770 immunotherapy., Proc. Natl. Acad. Sci. U. S. A. 106 (2009) 5497-502.

$771 \quad$ doi:10.1073/pnas.0901592106.

772 [34] E.M. Bachelder, T.T. Beaudette, K.E. Broaders, J. Dashe, J.M.J. Fréchet, Acetal773 derivatized dextran: An acid-responsive biodegradable material for therapeutic applications, J. Am. Chem. Soc. 130 (2008) 10494-10495. doi:10.1021/ja803947s.

[35] S. a Meenach, Y.J. Kim, K.J. Kauffman, N. Kanthamneni, E.M. Bachelder, K.M. Ainslie, Synthesis, Optimization, and Characterization of Camptothecin- Loaded Acetalated Dextran Porous Microparticles for Pulmonary Delivery, Mol Pharm. (2012). doi:10.1021/mp2003785.

780

[36] K.J. Peine, M. Guerau-De-Arellano, P. Lee, N. Kanthamneni, M. Severin, G.D. Probst, H. Peng, Y. Yang, Z. Vangundy, T.L. Papenfuss, A.E. Lovett-Racke, E.M. Bachelder, K.M. Ainslie, Treatment of experimental autoimmune encephalomyelitis by codelivery of disease associated peptide and dexamethasone in acetalated dextran microparticles, Mol. Pharm. 11 (2014) 828-835. doi:10.1021/mp4005172.

[37] Z. Wang, S.A. Meenach, Synthesis and Characterization of Nanocomposite Microparticles (nCmP) for the Treatment of Cystic Fibrosis-Related Infections, Pharm. Res. (2016) 1-11. doi:10.1007/s11095-016-1921-5. sensitive nanoparticles for colonic delivery of curcumin in inflammatory bowel 
disease, Int. J. Pharm. 473 (2014) 203-212. doi:10.1016/j.jpharm.2014.07.009.

791 [39] A. Kunwar, A. Barik, B. Mishra, K. Rathinasamy, R. Pandey, K.I. Priyadarsini,

792 Quantitative cellular uptake, localization and cytotoxicity of curcumin in normal

793 and tumor cells, Biochim. Biophys. Acta - Gen. Subj. 1780 (2008) 673-679.

$794 \quad$ doi:10.1016/j.bbagen.2007.11.016.

795 [40] Y. Cong, L. Wang, A. Konrad, T. Schoeb, C.O. Elson, Curcumin induces the 796 tolerogenic dendritic cell that promotes differentiation of intestine-protective regulatory T cells, Eur. J. Immunol. 39 (2009) 3134-3146. doi:10.1002/eji.200939052.

799 [41] S. Chanburee, W. Tiyaboonchai, Enhanced intestinal absorption of curcumin in 800 Caco-2 cell monolayer using mucoadhesive nanostructured lipid carriers., J.

801 Biomed. Mater. Res. B. Appl. Biomater. (2017) 1-8. doi:10.1002/jbm.b.33884.

802 [42] S. Tima, S. Anuchapreeda, C. Ampasavate, C. Berkland, S. Okonogi, Stable 803 curcumin-loaded polymeric micellar formulation for enhancing cellular uptake and 804 cytotoxicity to FLT3 overexpressing EoL-1 leukemic cells, Eur. J. Pharm.

$805 \quad$ Biopharm. 114 (2017) 57-68. doi:10.1016/j.ejpb.2016.12.032.

806 [43] S.A. Malekar, A.L. Sarode, A.C. Bach, A. Bose, G. Bothun, D.R. Worthen, Radio 807 Frequency-Activated Nanoliposomes for Controlled Combination Drug Delivery., 808 AAPS PharmSciTech. 16 (2015) 1335-43. doi:10.1208/s12249-015-0323-z.

809 [44] S.A. Malekar, A.L. Sarode, A.C. Bach, D.R. Worthen, The Localization of 810 Phenolic Compounds in Liposomal Bilayers and Their Effects on Surface 811 Characteristics and Colloidal Stability., AAPS PharmSciTech. 17 (2016) in press. 812 doi:10.1208/s12249-016-0483-5. 
813 [45] L. Procházková, Y. Rodríguez-Muñoz, J. Procházka, J. Wanner, Simple

814 spectrophotometric method for determination of polyvinylalcohol in different

815 types of wastewater, Int. J. Environ. Anal. Chem. 94 (2014) 399-410.

816 doi:10.1080/03067319.2013.853761.

817 [46] J. Charles, M. Stewart, Colorimetric Determination of Phospholipids with

818 Ammonium Ferrothiocyanate, Anal. Biochem. 14 (1980) 10-14.

819 doi:10.1016/0003-2697(80)90269-9.

820 [47] J.W. Coleman, Nitric Oxide in Immunity and Inflammation, Int.

821 Immunopharmacol. 1 (2001) 1397-1406. doi:10.1016/j.neuroscience.2018.06.012.

822 [48] S.E.A. Gratton, P.A. Ropp, P.D. Pohlhaus, J.C. Luft, V.J. Madden, M.E. Napier,

823 J.M. Desimone, The effect of particle design on cellular internalization pathways, 824 Proc Natl Acad Sci U S A. 105 (2008).

825 [49] A. Albanese, P.S. Tang, W.C.W. Chan, The Effect of Nanoparticle Size, Shape, 826 and Surface Chemistry on Biological Systems, Annu. Rev. Biomed. Eng. (2012). 827 doi:10.1146/annurev-bioeng-071811-150124.

828 [50] D.M.K. Jensen, D. Cun, M.J. Maltesen, S. Frokjaer, H.M. Nielsen, C. Foged, 829 Spray drying of siRNA-containing PLGA nanoparticles intended for inhalation, J.

$830 \quad$ Control. Release. 142 (2010) 138-145. doi:10.1016/j.jconrel.2009.10.010.

831 [51] S. Honary, F. Zahir, Effect of Zeta Potential on the Properties of Nano - Drug

832 Delivery Systems - A Review (Part 2), Trop. J. Pharm. Al Res. 12 (2013) 265-273.

833 doi:10.4314/tjpr.v12i2.19.

834 [52] S. Honary, F. Zahir, Effect of zeta potential on the properties of nano-drug

835 delivery systems - A review (Part 1), Trop. J. Pharm. Res. 12 (2013) 255-264. 
doi:10.4314/tjpr.v12i2.19.

837 [53] J.G. Weers, D.P. Miller, Formulation Design of Dry Powders for Inhalation, J.

838 Pharm. Sci. 104 (2015) 3259-3288. doi:10.1002/jps.24574.

839 [54] R.P. Barroso, L.G.M. Basso, A.J. Costa-filho, Interactions of the antimalarial

840 amodiaquine with lipid model membranes, Chem. Phys. Lipids. 186 (2015) 68-78.

$841 \quad$ doi:10.1016/j.chemphyslip.2014.12.003.

842 [55] R.H. Fang, S. Aryal, C.M.J. Hu, L. Zhang, Quick synthesis of lipid-polymer

843 hybrid nanoparticles with low polydispersity using a single-step sonication

844 method, Langmuir. 26 (2010) 16958-16962. doi:10.1021/la103576a.

845 [56] E. Maretti, T. Rossi, M. Bondi, M.A. Croce, M. Hanuskova, E. Leo, F. Sacchetti,

846 V. Iannuccelli, Inhaled Solid Lipid Microparticles to target alveolar macrophages

847 for tuberculosis, Int. J. Pharm. 462 (2014) 74-82.

$848 \quad$ doi:10.1016/j.ijpharm.2013.12.034.

849 